\title{
Leidenfrost evaporation of water droplet
}

\author{
Tadeusz Orzechowski* \\ Kielce University of Technology, Al. Tysiąclecia PP 7, 25-314 Kielce, Poland
}

\begin{abstract}
The present paper examines the behaviour of a single large droplet levitating over a hot surface, unsteady mass of the droplet, and heat transfer. It was assumed that the evaporation from the upper surface of the droplet is negligibly small compared with the amount discharged from its lower surface, and the heat transfer coefficient is the power function of droplet orthogonal projection onto the heating surface. Based on the photographic documentation, the dependence of the droplet projection on time was approximated. A heat balance was written in the form of a non-linear first order differential equation. The solution to the equation was included. The analytical function of droplet mass change in time was used to determine the exponent of the power dependence of the heat transfer coefficient on the orthogonal droplet projection onto the heating surface. The comparison showed the method proposed in the study could be applied to analyse the behaviour of a water drop levitating above a surface at the temperature higher than the Leidenfrost point.
\end{abstract}

\section{Introduction}

In the mid-1980s, the thermal load on components amounted to $100 \mathrm{~W} / \mathrm{cm}^{2}$ [1]. The only possible solution was to use cooling that employed change of phase processes in the cooling agent $[2,3,4]$. Another important application area of phase-change cooling includes evaporation processes in refrigeration and air conditioning $[5,6]$. In thermal treatment of metals, controlled temperature changes are decisive for the formation of near-surface microstructures [7].

Surface spray cooling is another important issue [8]. Various investigations conducted into the subject focus on the physics of the phenomenon, which depends strongly on the liquid and its components [9] and [10], thermal and flow conditions [11,12], interaction with the surface [13], radiation heat transmission through the water film [14], etc. Also, when the basic liquid is modified with additives such as nanoparticles, significant changes in the nature of heat transfer are observed [15]. A brief review of their impact on the Leidenfrost temperature in heat transfer applications is delivered in [16].

Physical processes involved in droplet evaporation are closely related to the surface. It was shown that droplets located on low conductivity materials get colder and evaporate at about half the rate of similar droplets on surfaces with high thermal conductivity [17]. The evaporation characteristics of sessile water droplets on smooth hydrophobic and structured superhydrophobic heated surfaces were experimentally investigated in [18]. It was proved that surfaces with high wettability enhance heat transfer coefficient and critical heat flux in pool boiling [19]. Clear differences are observed between nucleate pool boiling and boiling in the droplet. Intense nucleate boiling in the droplet is accompanied by continuous changes in its shape and the area of contact with the substrate. Those phenomena are caused by vapour bubbles that flow inside and depart. In this case, local changes in the surface temperature are observed, which results in a lower value of the heat transfer coefficient [20]. Only at the wall superheat above $10 \mathrm{~K}$, bubbles visibly change the droplet circumference. As a result, the surface is locally dried up, and the density of the removed heat is considerably reduced. Surface temperature grows and the inflowing liquid evaporates abruptly, which makes the whole process unstable. This range is termed a transition boiling regime [21]. The final stage of this regime is the formation of a stable vapour film, which supports the droplet above the heating surface [22,23], while intense internal currents inside the droplet are observed [24]. The first observation of that effect was reported by Johann Gottlob Leidenfrost in A Tract About Some Qualities of Common Water in 1756, and the phenomenon has been named after him.

In many technological processes, the element that needs to be cooled has the initial temperature that is higher than Leidenfrost temperature. The amount of heat carried away and the rate of cooling are the parameters that need to be precisely controlled [25]. That refers to technological processes [26,27], and to maintaining the thermal stability of the elements cooled [28]. To solve the task thus posed, it is necessary to have possibly complete information on the physical processes that occur in droplet evaporation, which despite numerous investigations have not been fully explained.

The aim of this study is to provide the analysis of heat transfer between a large water droplet at atmospheric pressure, and a hot surface at a temperature higher than Leidenfrost temperature.

\footnotetext{
*Corresponding author: todek@tu.kielce.pl
} 


\section{Experimental set-up}

A large droplet of water was placed on the heating surface with temperature higher than the Leidenfrost point. Measurements taken for this study concerned the droplet mass changes in time. Electronic weighing scales, with the maximum capacity of $500 \mathrm{~g}$, was used. The scales readability is $0.02 \mathrm{~g}$, and sensitivity is 0.001 $\mathrm{g}$. The interface is used for direct communication with the computer.
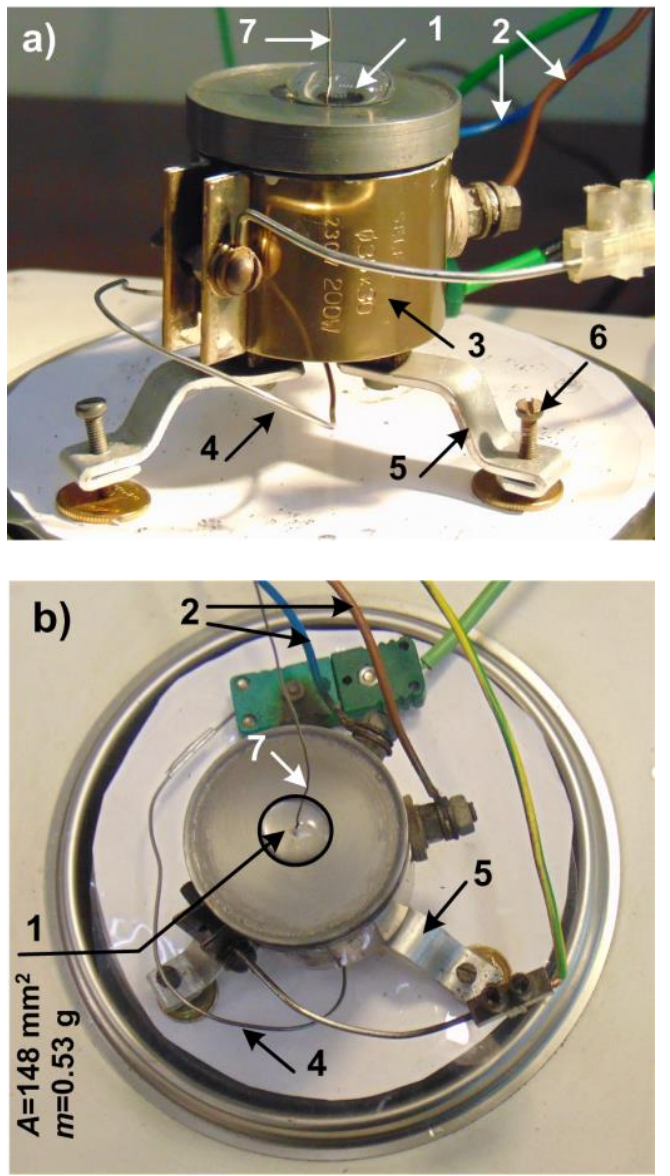

Fig. 1. Photograph of the experimental set-up: a) side view, b) top view 1- droplet, 2 - electrical power supply, 3 - heater, 4 thermocouple, 5- support pin, 6 - screw, 7 - thermocouple.

Investigations into droplet evaporation at a temperature above the Leidenfrost point were conducted at the stand, the main component of which was a cylindrical copper heater (see Fig. 1). The cylinder upper surface was shaped like a bowl having a very large radius, which allowed centric, stable positioning of the liquid droplet under investigation, as shown in Fig.1b. Directly under the surface, in the symmetry axis, k-type sheathed thermocouple was placed. The heater was installed on the cylinder outer surface and supplied with electric power from the autotransformer by suspended conductors. The output power of the heater was set by voltage, adjusted according to the voltmeter readings. The detailed description of the measurement procedure, together with the analysis of uncertainties were presented in [29] and [30].

\section{Results and discussion}

Large mass droplets show instabilities of various types, which result from complex convective movements inside the droplet [31-34].

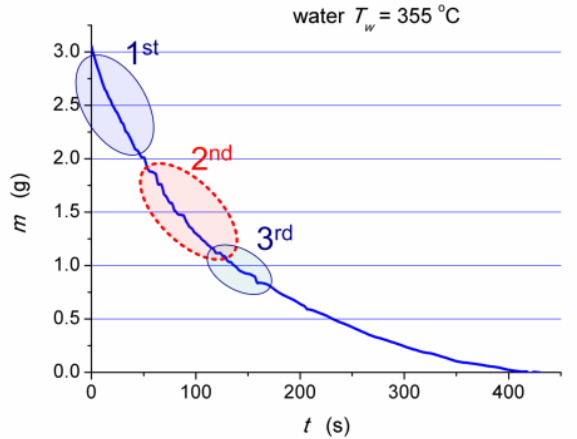

Fig. 2. Evaporation of the water droplet on the wall of the temperature $T_{w}=355^{\circ} \mathrm{C}$.

In Fig.2, three zones with different droplet behaviour are marked. In the first zone under the surface of the droplet having the mass of as much as approx. $2 \mathrm{~g}$, vapour lingers forming large bubbles. They grow very slowly and relatively seldom depart freely through the droplet upper surface.
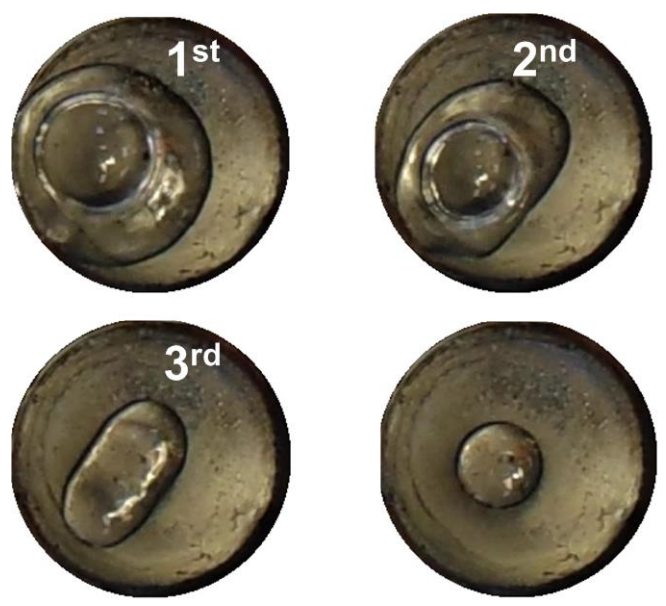

Fig. 3. Droplet shape evolution at various stages of its evaporation acc. Fig. 2.

Due to the measurement system inertia, relatively small changes in weight are recorded during this stage. As the droplet mass decreases, the area of its surface is also reduced, which leads to the deterioration of vapour accumulation. The frequency of bubble departure clearly rises, which is reflected in the readings of the scales in the second zone. For droplets having the mass of approx. $1 \mathrm{~g}$, vapour bubbles departing from the upper surface are not observed. Small changes in the droplet weight in third zone result from a chaotic droplet movement over the surface. Small drops weighing less than $0.7 \mathrm{~g}$ take on stable shapes, as shown in Figure 3.

Photographic imaging makes it possible to measure droplet projection onto the heating surface. Digital photo camera was suspended directly above the droplet to record the projection of its surface. Then, the footage was converted into individual frames. Using software 
tools, droplet surface area was estimated in the selected frames. For the series of concern, images are presented in Fig.4.

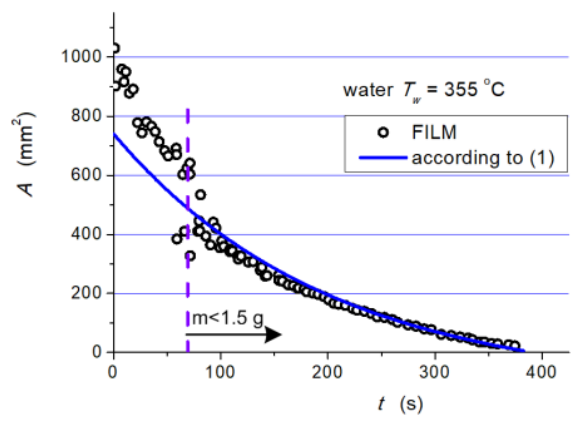

Fig. 4. Droplet projection onto the heating surface.

The literature provides different formulas for converting droplet orthogonal projection onto the heating surface. The correlations are usually time-dependent power formulas. One of them has the following form [35]:

$$
\frac{A(t)}{A_{0}}=C_{B}\left(a^{t}-C_{A}\right)
$$

where $A_{0}$ is the surface projection at the initial instant, whereas $C_{A}, C_{A}>0$ and $a<1$ are constants under the assumed and pre-set conditions of the experiment.

Using the correlation above (1), droplet surface area was calculated as a function of time, as shown in Fig.4. The fitting of the curve from Eq. (1) was conducted in such a way so that it could be matched, as accurately as possible, to the results obtained from photographic imaging. The comparison of the profiles of both curves shows fairly good congruence for small mass droplets. For large mass droplets, i.e. above $1.5 \mathrm{~g}$, a clear discrepancy between the curves is observed.

\section{Heat transfer analysis}

Assuming that evaporation from the upper surface of the droplet is small compared with the amount of heat carried away from the bottom surface, the overall heat transfer coefficient comprises the convective and radiative part, i.e. $\alpha=\alpha_{c}+\alpha_{r}$. Then, it possible to write energy balance equation, which implies a change in droplet mass $m$ in time $t$ :

$$
\alpha\left(T_{w}-T_{d}\right) A=-\left(c_{p}\left(T_{s}-T_{d}\right)+h_{f g}\right) \frac{\mathrm{d} m}{\mathrm{~d} t}
$$

where $T_{w}, T_{d}$ and $T_{s}$ are surface, droplet and saturation temperatures respectively, $A-$ orthogonal droplet projection onto the heating surface, $c_{p}$ - specific heat, and $h_{f g}$ phase change enthalpy at saturation temperature, here at atmospheric pressure.

To obtain solution to Eq. (2), it is necessary to provide a correlation for droplet projection onto the heating surface and heat transfer coefficient. Experimental data indicate exponential dependence of heat transfer coefficient on the surface. For that reason, the following form of the function was assumed [30]:

$$
\alpha=C_{\theta} A^{n}
$$

where $n$ is a constant exponent, and coefficient $C_{\theta}=C\left(T_{w}\right)$ is different for individual measurement curves, and varies with the wall temperature $T_{w}$.

The equation (2) together with the adopted assumptions (1) and (3) can be integrated, and its solution has the following form:

$$
\begin{aligned}
& m_{0}-m=M_{0}\left(C_{B} C_{A}\right)^{n+1} \frac{1}{\ln a} * \\
& *\left[\int \frac{\left.(z-1)^{n+1} d z-\left.\int \frac{(z-1)^{n+1}}{z} d z\right|_{t=0 \rightarrow z=1 / C_{A}}\right]}{z}\right]
\end{aligned}
$$

where for the sake of convenience, a new variable is introduced:

$$
z=\frac{a^{t}}{C_{A}}
$$

At $t=0$, droplet mass $m_{0}$ is known and $M_{0}$ is a flux of mass evaporated from the droplet surface at $t=0$ defined as follows:

$$
M_{0}=\frac{T_{w}-T_{d}}{h_{f g}+c_{p}\left(T_{s}-T_{d}\right)} C_{\theta}\left(A_{0}\right)^{n+1}
$$

The integral in the equation above can be presented as an infinite series:

$$
\begin{aligned}
& \int \frac{(z-1)^{n+1}}{z} d z=\sum_{i=1}^{j} \frac{(i-1) !}{\sum_{k=1}^{i}(n+1+k)} \frac{(z-1)^{n+1+i}}{z^{i}}+ \\
& +\frac{(j-1) !}{(n+2)(n+3) \ldots(n+1+j)} \int \frac{(z-1)^{n+j}}{z^{j+1}} d z
\end{aligned}
$$

which is a fast-converging series. The first term of the sum on the right-hand side of the equation (7) is a partial sum, whereas the second term is the series remainder. A solution with accuracy below $1 \%$ is obtained already when only ten terms are taken into account, i.e. $j \leq 10$.

Shape disturbances essentially alter droplet projection onto the heating surface, which can be seen in Figs. 3 and 4. The disturbances are caused by lingering and successive departing of large vapour bubbles. Therefore, further analysis was limited to droplets having a mass of $m \leq 1.5 \mathrm{~g}$. Using correlation (1) for orthogonal projection vs. time, the surface area of the droplet was calculated, as shown in Fig.4.

The following values of coefficients were assumed: $C_{A}=0.11, C_{B}=1.16$, and $a=0.9943 . C_{A}$ and $C_{B}$ were calculated from the dependence given in [35]. The base of power was selected following the criterion of the 
minimum mean quadratic error between the measured areas and areas computed acc. Eq. (1).

The accuracy of the approximation can be evaluated by comparing residuals between measurement points and calculated points at the same time instant. The greatest differences between computed and measured quantities are found for the initial period for small time values, where clear spherical shape aberration is visible, as can be seen in Fig. 3.

The solution (4) to the problem of concern determines the dependence of the droplet mass on time. To plot the curve, all the coefficients used before are needed. Three of them, namely $C_{A}, C_{B}$ and $a$, result from the fitting of the correlation for droplet orthogonal projection on the heating surface. The remaining quantities that need to be specified are coefficient $C_{\theta}$ and power exponent $n$. They are related to the heat transfer coefficient, the magnitude of which is proposed to be expressed by power dependence on the instantaneous droplet projection area $A$. The values of both quantities mentioned above are decisive for magnitude of the mass flux that is evaporated. Therefore, they need to be selected in such a way so that the curve of droplet mass change in time, calculated acc. Eq. (4), would closely coincide with the curve determined experimentally, as shown in Fig.5.

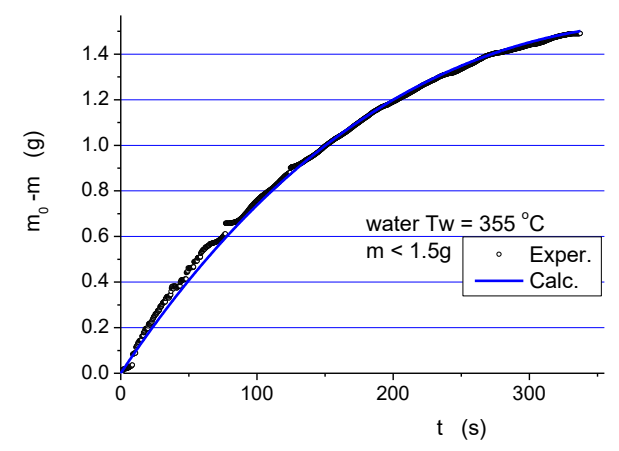

Fig. 5. Measured and calculated instantaneous droplet mass.

Both coefficients were determined acc. data from Fig. 5 following the criterion of the minimum mean quadratic error. Approximated values $C_{\theta}=19.1$ and $n=-$ 0.3 make it possible to calculate local values of the heat transfer coefficient acc. formula (3), as shown in Fig. 6.

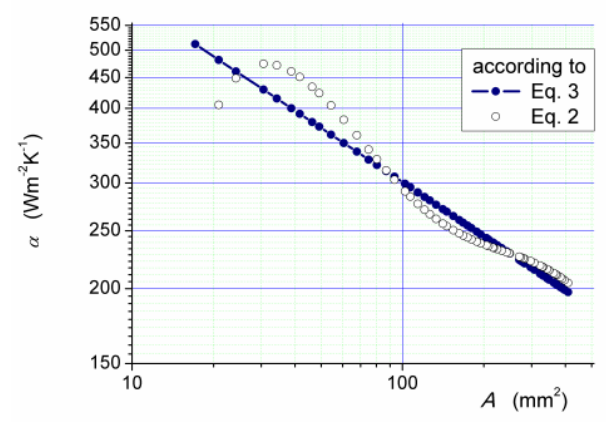

Fig. 6. Local values of the coefficient of heat transfer from the bottom water droplet surface for the orthogonal projections onto the heating surface at constant temperature $T_{w}=355^{\circ} \mathrm{C}$.
For the sake of comparison, the results of direct calculations from Eq. (2) are also given in that figure. The computational procedure was discussed in study [30].

\section{Conclsions}

A large droplet of water was placed on the heating surface with temperature higher than the Leidenfrost point. The analysis of results was carried out employing the droplet heat balance, under the assumption that heat transfer results only from droplet interaction with the heating surface. To make calculations, the dependence, available in the literature, for the droplet orthogonal projection as the function of time was applied (see Eq. 1). On the basis of the author's investigations and photographic imaging the following values were selected: coefficients $C_{A}=0.11, C_{B}=1.16$, and also $a=0.9943$. The values were calculated for the droplet with mass below $1.5 \mathrm{~g}$ and heating surface temperature $T_{w}=355^{\circ} \mathrm{C}$ at the condition of the minimum mean quadratic error.

The comparison of the measured values of mass loss in time and the computed ones made it possible to determine coefficients $C_{\theta}$ and $n$. They specify instantaneous values of the heat transfer coefficient acc. Eq. (3). They were determined on the basis of the data in Fig. 5 for the criterion of the minimal mean quadratic error.

The determined values $C_{\theta}=19.1$ and $n=-0.3$ make it possible to compute local values of the heat transfer coefficients acc. formula (3), which was shown in Fig. 6. For the sake of comparison, the results of direct computations acc. Eq. (2) were also given in the figure. In the procedure, polynomial approximation of the mass flux carried away from the droplet surface is used. That causes fluctuation visible in Fig. 6, which is difficult to eliminate, especially at the boundaries of the region. When analytical solution (Eq. 4) is applied, this inconvenience can be avoided. Also, the adopted power dependence of instantaneous values of the heat transfer coefficient on droplet orthogonal projection onto the heating surface is confirmed.

\section{References}

1. M. Aamir, Q. Liao, W. Hong, Z. Xun, S. Song, M. Sajid, Heat Mass Transfer, 53 (2017)

2. S. Hożejowska, J. Theor. Appl. Mech. 53(4) (2015)

3. T. Orzechowski, Heat Mass Transfer, 52 (2016)

4. T. Orzechowski, EPJ Web of Conferences, 143 (2017)

5. T. Orzechowski, Z. Skrobacki, EPJ Web of Conferences, 114 (2016)

6. P.O. Rašković, G.D. Vučković, M.V. Vukić, Therm. Science, 12 (4) (2018)

7. K.H.M. Abdalrahman, S. Sabariman, E. Specht, Int. J. Heat Mass Tran. 78 (2014)

8. M. Gradeck, N. Seiler, P. Ruyer, D. Maillet, Exp. Therm. Fluid Sci. 47 (2013) 
9. P. Keller, A. Bader, C. Hasse, Int. J. Heat Mass Tran. 67 (2013)

10. Y. Wang, Y. Jiang, W. Chen, B. Zhou, Appl. Therm. Eng. 123 (2017)

11. V.E. Nakoryakov, S.Y. Misyura, S.L. Elistratov, Int. J. Heat Mass Tran. 55 (2012)

12. K. Strąk, M. Piasecka, B. Maciejewska, Int. J. Heat Mass Tran. 117 (2017)

13. M. Gao, P. Kong, L-X. Zhang, J-N. Liu, Int. Commun. Heat Mass Tran. 88 (2017)

14. G.V. Kuznetsov, K.Y. Osipov, M.V. Piskunov, R.S. Volkov, Int. J. Heat Mass Tran. 117 (2018)

15. A.M. Ali, W. Arshad, H.M. Ali, H. M. Ali, M.A. Nasir, J. Thermal Science, 21(5) (2017)

16. S. Wciślik, Chem. Pap. 71 (2017)

17. T. Log, Appl. Therm. Eng. 93 (2016)

18. S. Dash, S.V. Garimella, Phys. Rev. E, 89 (2014)

19. X. Chen, H. Qiu, Int. J. Heat Mass Tran. 88 (2015)

20. Y. Wang, H. Fu, Q. Huang, Y. Cui, Y. Sun, L. Jiang, Energy, 93 (2015)

21. Y. Mitsutake, S. Illias, K. Tsubaki, M.N. Hasan, M. Monde, Procedia Eng. 105 (2015)

22. T. Orzechowski, EPJ Web of Conferences, 143 (2017)

23. T. Orzechowski, S. Wciślik, Exp. Therm. Fluid Sci. 59 (2014)

24. A. Al-Sharafi, B.S. Yilbas, H. Ali, Appl. Therm. Eng. 128 (2018)

25. Y.Y. Puzina, J. Phys. Conf. Ser. 891 (2017)

26. N. Shahidzadeh-Bonn, S. Rafa, A. Azouni1, D. Bonn, J. Fluid Mech. 549 (2006)

27. X. Liu, J. Liu, R. Xue, L. Chen, Y. Hou, Y, Exp. Therm. Fluid Sci. 92 (2018)

28. D. Liu, F-Y. Zhao, H-X. Yang, G-G. Tang, Energy, 83 (2015)

29. T. Orzechowski, T, S. Wciślik, Energ. Convers. Manage. 76 (2013)

30. T. Orzechowski, T, S. Wciślik, Int. J. Heat Mass Tran. 73 (2014)

31. D. Brutin, B. Sobac, F. Rigollet, C. Le Niliot, Exp. Therm. Fluid Sci. 35 (2011)

32. M. He, H. Qiu, H, Int. J. Therm. Sci. 100 (2016)

33. S. Wciślik, J. Phys.: Conf Ser, 745 (2016)

34. R. Kaniowski, R. Pastuszko, EPJ Web of Conf. 143, 02050 (2016)

35. G. Paul, P.K. Das, I. Manna, Exp. Therm. Fluid Sci. 60 (2015) 